\title{
Quiste pericárdico gigante Reporte de un caso
}

Jiménez-Serrano José Adrián*, Jiménez-González Adrián"**, Esparza-Pantoja Javier**, López-Viramontes Beatriz ${ }^{* * * *}$

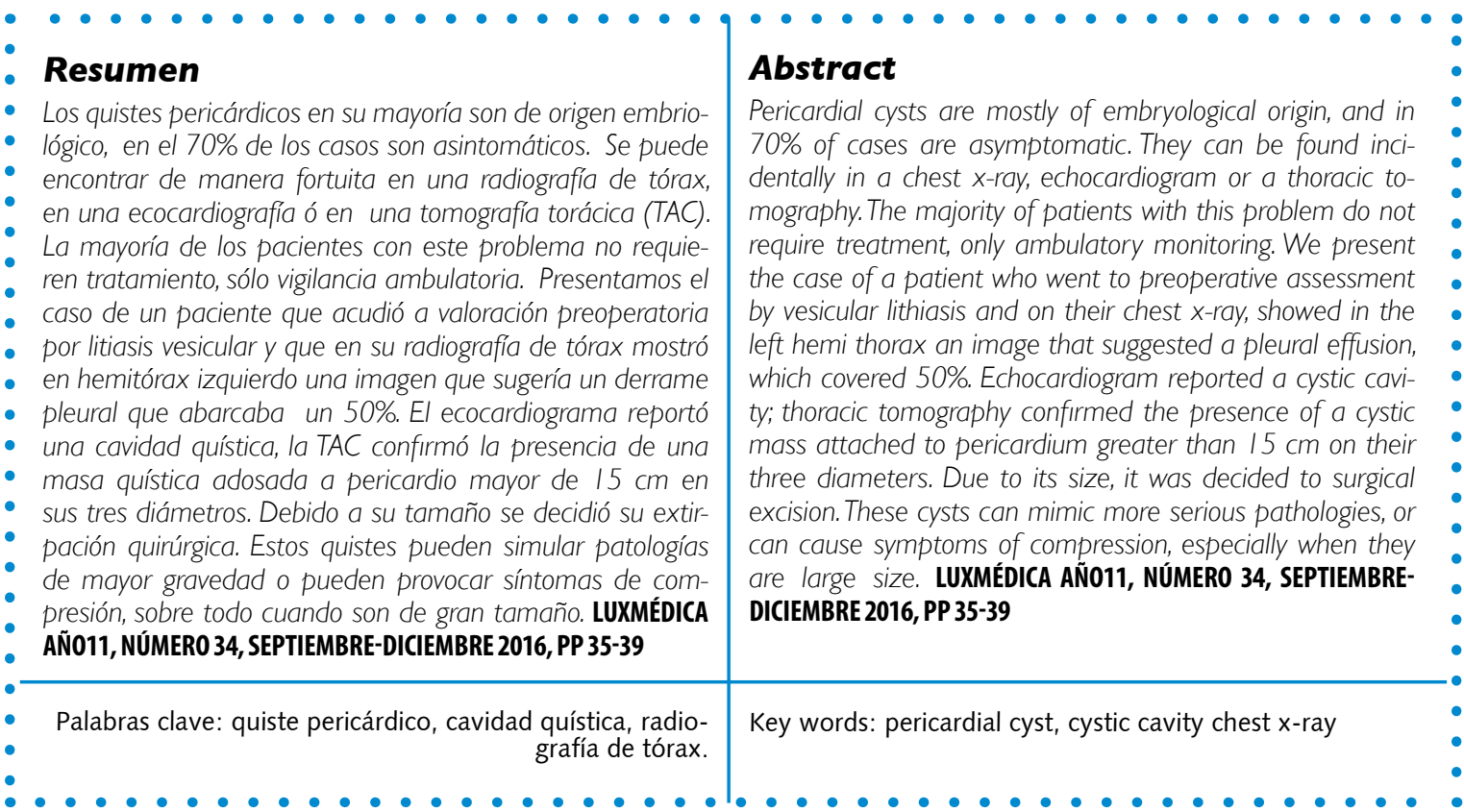

\section{Introducción}

Los quistes pericárdicos (QP) son una patología muy rara, representa el $7 \%$ de los tumores mediastínicos y el $20 \%$ de los de corazón y pericardio. ${ }^{1,2}$ El sitio de presentación más frecuente es en el ángulo cardiofrénico derecho. Por lo regular se descubren accidentalmente después de una radiografía de tórax, la cual se realizan por sintomatología no cardiogénica. Los quistes pericárdicos

* Cardiólogo. Hospital Starmédica. Aguascalientes, México. adrian.jimenez.uaa@gmail.com

** Médico Interno de Pregrado del Hospital General de Occidente, Guadalajara Jalisco, México kapiistrano@gmail.com

*** Cirujano de Corazón y Tórax. Hospital Starmédica, Aguascalientes, Ags, México zzzjepantoja@yahoo.com.mx

***** Cardióloga Ecocardiografista. Centro de estudios Cardiolab. Aguascalientes, Ags., México beatrizlopezv_1@yahoo.com.mx

Fecha de recibido: 13 de junio 2016

Fecha de aceptación: 13 de agosto 2016

Correspondencia:

Dr. José Adrián Jiménez Serrano. Av. Universidad 103-123, Villas de la Universidad, Aguascalientes, Ags. México. Código postal 20029 , teléfono (449)9129080, correo electrónico: adrian.jimenez.uaa@gmail.com 
pueden aparentar otras patologías, si el QP se localiza en el ángulo cardiofrénico derecho pueden sugerir dextrocardia, o bien derrame pleural cuando son gigantes. La relevancia de este caso se debe, al hallazgo de un quiste pericárdico gigante en el ángulo cardiofrénico izquierdo; localización poco frecuente, así como sus dimensiones que rebasan los $15 \mathrm{~cm}$ cuando normalmente miden de $2-5 \mathrm{~cm}$. El paciente acudió a valoración preoperatoria con diagnóstico de litiasis vesicular, asintomático al que se le encontró la anormalidad en la radiografía de tórax.

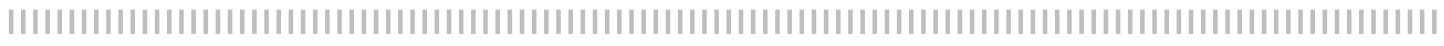

\section{Presentación del caso clínico}

Masculino de 67 años de edad, sin antecedentes de importancia, tabaquismo negativo, acude a valoración preoperatoria. No es hipertenso, no isquémico conocido, no refirió disnea ni ortopnea. A la exploración física, ruidos cardiacos rítmicos sin agregados, a la exploración de tórax se evidenció síndrome de derrame pleural izquierdo. Se pidió una radiografía de tórax, la cual mostró derrame pleural izquierdo que abarcaba un 50\% del hemitórax y sugería cardiomegalia global (figura 1). Llamó la atención que el paciente estuviera asintomático con ese grado de cardiomegalia y de derrame. El ecocardiograma encontró las 4 cavidades de tamaño normal y una fracción de expulsión de 64\%, además una imagen quística a la izquierda del corazón (figura 2). La tomografía de tórax reportó una masa quística en base de pulmón izquierdo con diámetros de más de $15 \mathrm{~cm}$ en sus tres dimensiones (figura 3). Se decidió extirpar dicho quiste, el cual midió $14 \mathrm{~cm} \times 15.4 \mathrm{~cm} \times 15.1 \mathrm{~cm}$ (figura 4) que contenía líquido tipo "agua de manantial" típico de los quistes pericárdicos.

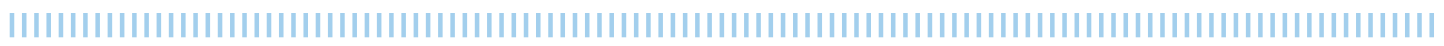

\section{Discusión}

Los primeros reportes de quiste pericárdico fueron a mediados del siglo XIX, cuando los patólogos encontraron en autopsias los hallazgos característicos de esta patología. La primera extracción quirúrgica de realizada en 1931 por Otto Pickhardt en el Lenox Hill Hospital de Nueva York. En 1943 Greenfield y su grupo de colaboradores la bautizaron como el quiste de "agua de manantial". ${ }^{1}$

La mayoría de los quistes pericárdicos tiene un origen congénito; por una falla en la embriogénesis de la cavidad celó- mica. ${ }^{2}$ Dentro de las causas adquiridas se han reportado, los de origen traumático, postquirúrgico de cirugía de corazón e inflamatorio por pericarditis reumática, infección por tuberculosis y equinococo; este último agente infeccioso origina el quiste hidatídico que generalmente se asocia con quistes en miocardio o en cualquier otro lugar del cuerpo. ${ }^{1} \mathrm{El}$ quiste hidatídico es extremadamente raro cuando se encuentra de manera aislada en pericardio. $^{3}$

Las lesiones quísticas en pericardio son una entidad muy rara que representa el 
$7 \%$ de tumores del mediastino y el $33 \%$ de los quistes mediastinales. ${ }^{1}$ La incidencia del quiste pericárdico se ha estimado en 1 por 100.000 casos. No se han reportado diferencias entre sexos. ${ }^{4}$ Son diagnosticados más frecuentemente desde la tercera década de la vida en adelante. ${ }^{5}$ La forma más común es unilocular pero pueden existir formas multiloculares debido a la presencia de bandas fibrosas constrictivas. ${ }^{6}$ La localización más común del quiste pericárdico es el ángulo cardiofrénico derecho en el $70 \%$ de los casos, mientras que en el izquierdo su frecuencia es del $22 \%$. Han sido reportados en el mediastino posterior, región hiliar derecha e izquierda, área paratraqueal derecha y arco aórtico. ${ }^{6,7}$ Su diámetro normalmente se encuentra entre $1 \mathrm{~cm}$ a $5 \mathrm{~cm}$, sin embargo, pueden llegar a ser más grandes. ${ }^{8}$

Histológicamente, la pared del quiste pericárdico está compuesta de tejido fibroso rodeado por mesotelio con datos de inflamación crónica, en su interior se encuentra un fluido transparente razón por la que en un principio se le llamó quiste de "agua de manantial".

Los pacientes con quiste pericárdico usualmente son asintomáticos (en el $60-75 \%$ de los casos). ${ }^{1}$ Cuando el quiste comprime estructuras vecinas aparecen los síntomas como tos crónica, dolor torácico, disnea, hemoptisis, fiebre, neumotórax ${ }^{7}$ y la sensación de opresión esternal. Son raros el síncope, neumonía, insuficiencia cardiaca congestiva y la muerte cardiaca súbita. ${ }^{9,10}$ Los quistes pericárdicos se pueden descubrir incidentalmente en una radiografía de tórax como una lesión ovalada homogénea adyacente al corazón. Al momento del descubrimiento se necesitan más estudios de imagen como la tomografía axial computarizada (TAC), ecocardiograma transtorácico y en ocasiones resonancia magnética (MRI). La TAC es considerada como la mejor herramienta para diagnóstico y seguimiento. ${ }^{1}$ Los quistes pericárdicos se observan como una masa oval homogénea, claramente definida con paredes delgadas y una densidad ligeramente mayor a la del agua. El medio de contraste intravenoso no es absorbido por el quiste pericárdico, así mismo su calcificación o rotura raramente ocurren. ${ }^{2}$ El ecocardiograma transtorácico tiene la ventaja de eliminar otros posibles diagnósticos como aneurisma de la aorta, grasa pericárdica prominente, tumores sólidos, orejuela izquierda prominente y aneurisma del ventrículo izquierdo. Se puede utilizar un ecocardiograma transesofágico como una herramienta clave para establecer el diagnóstico de quiste pericárdico en localizaciones atípicas. ${ }^{11}$ La aspiración del contenido del quiste puede servir como una herramienta de diagnóstico y tratamiento. ${ }^{6}$ El diagnóstico diferencial del quiste pericárdico se debe realizar con lipomas, angiomas, linfomas, carcinoma broncogénico, aneurismas del seno de Valsalva y hernia de Morgagni. ${ }^{4}$

El manejo del quiste pericárdico dependerá de su sintomatología. ${ }^{12}$ Algunos quistes desaparecen espontáneamente posterior a su ruptura dentro del espacio pleural. ${ }^{4}$ Si el paciente se encuentra asintomático; se puede realizar seguimiento mediante ecocardiograma secuencial. Si el paciente se encuentra sintomático o se observa un crecimiento del quiste o se encuentran componentes sólidos dentro del mismo, la resección quirúrgica mediante toracotomía o cirugía torácica asistida por video es el tratamiento más adecuado. Otra alternativa, es la aspiración del contenido de quiste, pero se ha reportado que el $30 \%$ de los pacientes presentan recurrencias a los tres años posteriores a la aspiración. La esclerosis con etanol ha demostrado una disminución de la recurrencia posterior a la aspiración. ${ }^{2}$ 


\section{Conclusiones}

Los quistes pericárdicos son una patología relativamente rara, representando solamente el $7 \%$ de las masas que se pueden encontrar en mediastino. Cuando encontramos en la radiografía de tórax un agrandamiento de la silueta cardiaca o que sugiere un derrame pleural como en este caso, debemos sospechar en un quiste pericárdico. Aunque generalmente son asintomáticos, es de suma importancia diagnosticarlos a tiempo, antes de que provoquen sintomatología debido a la compresión estructuras adyacentes. Su diagnóstico temprano puede ser difícil sólo con la radiografía, por lo que son de ayuda los paraclínicos ya comentados. Su tratamiento dependerá del tamaño, síntomas y patología concomitante.

\section{Bibliografía}

1. KAR SK GTDSMMBR. Pericardial Cyst: A Review of Historical Perspective and Currewnt Concept of Diagnosis and Management. ICJ. 2015; 1(1:8).

2. HEKMAT M GHTHSAMS. Giant Pericardical Cyst: A Case Report and Review of Literature. Iran J Radiol. 2016; 13(e21921).

3. TRAIBI A AFZAAAKE. Mediastinal Hydatid Cyst. J Chin Med Assoc. 2010; 73(3:7).

4. GERVILLA J SJMJDPMEOC. Quiste Pericárdico. Semergen. 2010; 36(174-176).

5. PORTILLO-CARROZ K MCEMR. Quiste Pleuropericárdico: Un Buen Final Para un Mal Inicio. Rev Esp Cardiol. 2006; 59(1079-83).

6. SALDAÑA C HA. Quiste Pericárdico Postraumático. An. Sist. Sanit. Navar. 2015; 38(475:478).

7. NAJIB M CHRAGLPPCR. Symptomatic Pericardial
Cyst: A Case Series. Ejechocard. 2011; 12(E43).

8. MAISCH B SPRAERRRAYTWTGYM. Guidelines on the Diagnosis and Management of Pericardical Diseases. EHJ. 2004; 02(1:28).

9. ILHAN E AFUOÖSKlea. An Unsual Presenttion of Pericardical Cys: Recurrent Syncope in a Young Patient. Cardiol J. 2012; 19(188-191).

10. FOROUZANDEH F KSBRALRMea. Giant Pericardical Cyst Presenting As Pneumonia. Tex Heart Inst J. 2012; 39(296-297).

11. BEZGIN T EAVSDCKAEA. Pericardial Cyst. Herz. 2014; 39(1005:1007)

12. HAMAD H GAAJVBBLFR. Giant Pericardial Cyst Mimicking Dextrocardia on Chest X-ray. Rev Port Cardiol. 2013; 32(49-52)

13. KAR SK GTDSMMVR. Pericardial Cyst: A Review of Historical Perspective and Current Concept of Diagnosis and Management. ICJ. 2015;: p. 1:8.

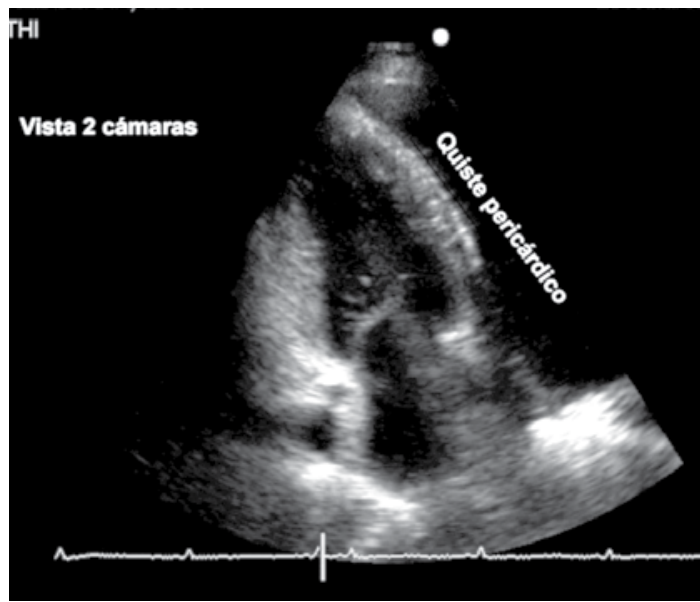

Fig. 2. Ecocardiograma, vista 2 camaras con imagen hipoecogénica de lado izquierdo del corazón.
Fig. 1. Radiografía de tórax que muestra

cardiomegalia e imagen que sugeria derrame pleural izquierdo.

.

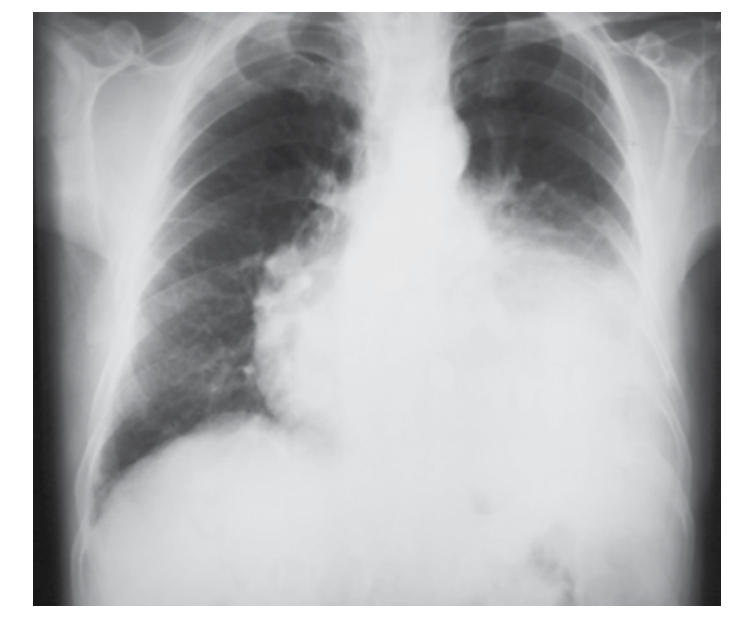




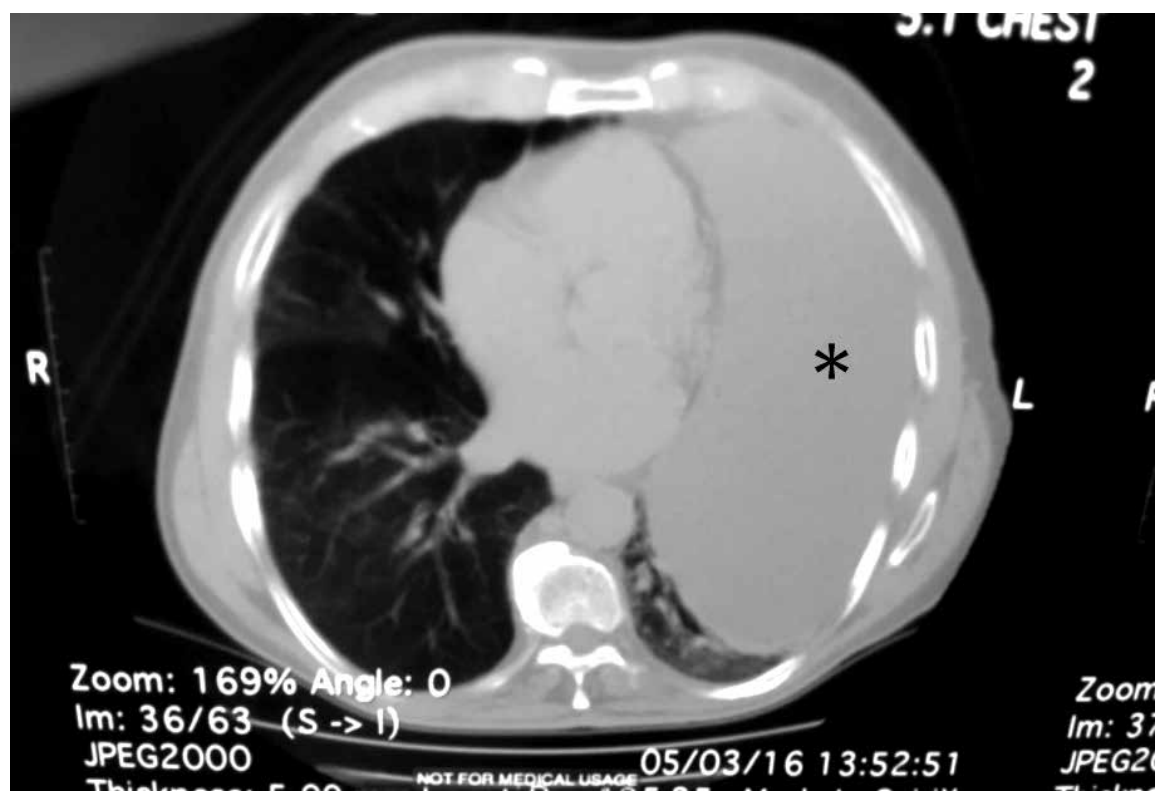

Fig 3. Imagen hipodensa que invade casi toda la base del hemitórax izquierdo *

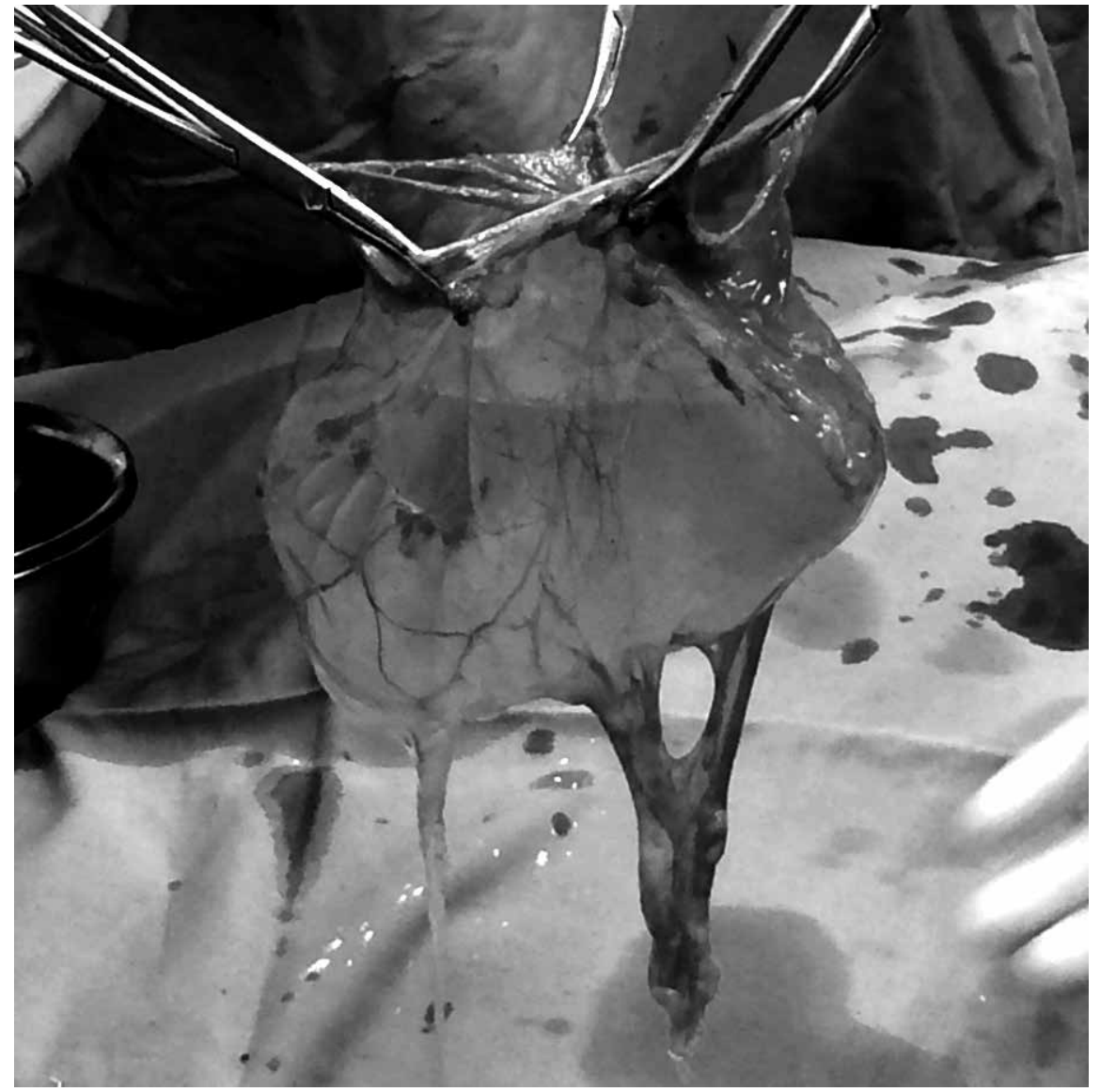

Fig. 4. Quiste pericardico, resecado por toracotomía izquierda. 